\title{
EL DISCERNIMIENTO APOSTÓLICO DE CARAA LA MISIÓN ECLESIAL DEL INSTITUTO RELIGIOSO
}

DOI: https://doi.org/10.52039/seminarios.v61i213.184

Germán ARANA BEORLEgUI

El discernimiento apostólico es aquel tipo de discreción espiritual que conduce a las opciones más adecuadas en la perspectiva del mayor fruto de la misión de nuestros Institutos. Este planteamiento sobre la naturaleza de nuestra misión y de su evangélica optimización no puede separarse de la fidelidad al propio carisma. Una fidelidad que no puede contentarse con reproducir de modo rutinario las mismas actividades, sino que, precisamente porque impulsada por el Espíritu es creativa y se descubre a sí misma de modo siempre nuevo en las cambiantes condiciones de la historia y de la cultura.

La orientación del gobierno adscrito a la jerarquía de la Iglesia por su naturaleza se circunscribe a los criterios más comunes y universales, para asegurar el bien común del Pueblo de Dios y su unidad expresada en las diversas situaciones de las Iglesias particulares. Sin embargo, es esencial a la misión apostólica de la Vida Consagrada (VC) su perfil carismático. Todo en la VC está marcado por el sello identitario del propio carisma. Porque en ella el ímpetu feraz del Evangelio se expresa en el conjunto de las multiformes concreciones de su radicalismo generadas por la potencia del único Espíritu. Potencia a la vez comunional y discriminante. Esto explica por qué la VC en la Iglesia no constituye un estamento uniforme, sino que subsiste sólo en sus singulares formas carismáticas, sea respecto de su estilo de vida y organización, sea respecto de su misión.

Ahora, sin sustraernos de esta perspectiva, podemos considerar la fuente del discernimiento espiritual para descender más adelante a sus concreciones.

\section{LA FUENTE DEL DISCERNIMIENTO ESPIRITUAL}

El discernimiento espiritual es un proceso en el que se entrecruzan dos vectores en dirección opuesta que finalmente se reconocen respectivamente. Por una parte la moción primigenia hacia el bien se genera en el corazón converti-

* Germán Arana es padre jesuita. Actualmente es rector y profesor en la Pontificia Universidad Comillas en Madrid, España. 
do. En el hontanar del corazón humano resuena la vivencia, cuya experiencia adquiere forma, timbre y color por emplear un símil musical. Es decir, adquiere una representación inteligente, vinculada a una modulación afectiva y a una carga volitiva. De aquí la importancia del aviso evangélico sobre la rectitud de intención, precisamente porque es el manantial que conforma y garantiza la orientación del hombre hacia el bien.

Sin embargo, el discernimiento no se sustrae de los acontecimientos externos, como recluido en un escenario exclusivamente interior. Como si éste fuera el resultado del descubrimiento de un universo idealístico que brota del arcano de la propia sensibilidad desligada de los acontecimientos en los que está insertada. El auténtico hombre interior no es un tipo que fija la brújula de su propósito, ignaro de cuanto sucede en derredor suyo. Es b ien conocido cuán infecundo resulta un voluntarismo empecinado al margen de una adecuada percepción de la realidad.

La lectura de la realidad es parte esencial del discernimiento no sólo porque asegura el modo, históricamente correspondiente a la voluntad de Dios, de actuar aquí y ahora, sino además porque contiene señales orientadoras acerca de la realización del proyecto divino. Es lo que llamamos los «signos de los tiempos». Jesús nos invita a discernir el tiempo presente (Lc 12, 56). El término griego kairós aquí empleado no indica simplemente el flujo cambiante de los acontecimientos, sino el compromiso de la libertad dispuesta a percibir el tiempo presente como oportunidad salvífica, como promesa ofrecida y gracia incoada. Dicho de otro modo, la realidad histórica atravesada de un sentido escatológico. En el modo de vivir aquí y ahora el cristiano su inserción en la historia se juega su suerte definitiva.

El estímulo que impulsa a la acción a veces es más interior, como una onda emergente que reformula el proyecto de vida del individuo. De esta guisa fue el primer discernimiento ignaciano en su larga postración en la casa de sus mayores herido de guerra. Otras veces surge más bien de un poderoso reclamo que impacta desde el exterior y solicita el corazón generoso del individuo hacia un compromiso mayor.

El discernimiento es una intuición del corazón convertido del creyente asimilado a la mentalidad de Nuestro Señor, que colige los signos salvíficos del tiempo presente. Se trata de una iluminación situada que nos lleva a un compromiso asimismo históricamente situado. La fe interpreta el presente históri$\mathrm{co}$, ya se muestre crudo o bonancible, y resuene con cualesquiera armónicos afectivos, como una oportunidad de realización amatoria, y como una promesa que la hace plausible. Esto implica que el modo de afrontar la hora presente del creyente lleva un sello estimulante e intrépido precisamente porque recibe el don que se le ofrece. El tono apagado y desilusionado de ciertos sujetos y de ciertas comunidades en su modo de percibirse insertas en el tiempo presente no revela solamente una psicología herida, ni es fruto solamente de las dificultades 
objetivas en las que se debaten, sino que tiene que ver con una fe empobrecida que oscurece la percepción de la gracia de Dios ofrecida en la hora presente.

La interacción armoniosa entre la propia interioridad y la realidad histórica se entiende mejor desde la perspectiva pedagógica del Evangelio. Jesús habría vislumbrado íntimamente la cercanía de la hora de partir de su casa para dedicarse de lleno a la misión de su Padre. Pero no poner fin a aquella suave convivencia con su madre sino cuando llega el reclamo del Bautista, que actúa como una señal desencadenante de la hora de su ministerio público. Más adelante Jesús, rodeado de sus discípulos, se mueve ciertamente con una precisa finalidad que surge de su voluntad salvífica, pero nunca ignorante ni despreocupada de la situación concreta de los que se le acercan. De tal suerte que se deja afectar por la llamada de las diversas formas de pobreza. Impelido a responder al momento también impedirá a los discípulos de desentenderse de tales urgencias modificando sus planes precedentes. Aquel «dadles vosotros de comer» (Mc 6, 37) resuena permanentemente como una poderosa llamada del Señor a su Iglesia para acudir de inmediato a las necesidades más lacerantes.

La dialéctica del discernimiento se entiende poniendo en el punto de mira el Corazón de Jesús. Acerca de ello me resulta fascinante rastrear en el Evangelio el lenguaje de la misericordia (oiktiro, eleeo, splagnidsomai), sobre todo el uso del verbo splagnidsomai ${ }^{1}$ propio de los sinópticos. Éste no es el único utilizado para expresar la capacidad de Jesús de dejarse afectar por el dolor de la enfermedad física o moral, de la soledad de los marginados, del retorcimiento del hambre, y finalmente del desgarro violento de la muerte. Con todo, me parece una expresión paradigmática de la moción compasiva de Jesús, ya sea por su intensidad afectiva, como por la puesta en marcha que desencadena de una acción reconstructora.

Este verbo tiene una forma gramatical pasiva. Como un movimiento de la sensibilidad, una forma de afección, que hace resonar interiormente lo que impresiona del exterior. Semánticamente apunta a la convulsión de las vísceras, de las entrañas humanas. En el lenguaje bíblico es una alegría somática para la sede más profunda de los sentimientos y el manantial de los deseos más ardientes. En nuestro lenguaje usamos otra alegoría somática: El corazón.

Esta expresión recoge la profunda resonancia en el corazón de Jesús del dolor humano y de su desamparo, y a la vez su capacidad propositiva y actual de resolver la insania. Un sentirse afectado de tal suerte que no se detiene en los armónicos afectivos, sino que se implica de inmediato en su resolución.

Con la diferencia que Jesús desplegaba su exousia, es decir, aquella autoridad suya propia del Verbo encarnado, de quen tiene la fuerza de Dios. Se trata de la prodigiosa capacidad del despertar del lecho de la muerte, de devolver a $15,20$.

1. Cf. Mt 9,$36 ; 14,14 ; 15,32 ; 18,27 ; 20,34 ;$ Mc 1, 41; 6, 34; 8, 2; 9, 22; Lc 7, 13; 10, 33; 
la comunidad a los malditos sociales, de practicar una medicina eficacísima de todas las especialidades, de restaurar una multitud hambrienta, de proponerse como guía segura a los extraviados, de aliviar lacerantes dolores. Una potencia transformante del hombre de tal alcance que llegaba a aquel asombroso imperativo, exclusivo de la autoridad divina: Tus pecados son perdonados ${ }^{2}$.

Sin embargo, Jesús no utilizaba su autoridad divina como una suerte de varita mágica para suscitar una admiración engañadora con respecto a su identidad. El Señor evitaba aparecer como un taumaturgo en el que pudieran concentrarse las apetencias desordenadas de beneficio inmediato dispensado a placer de la clientela. En su humanidad se escancia la caridad divina con rasgos particulares. Rasgos, en cierto sentido, contraculturales, o, al menos en alguna medida, inesperados.

Dicho en palabras sencillas, Jesús no actuaba de acaudalado que organiza una fundación de asistencia social sin que su vida regalada fuese mínimamente tocada; ni de charlatán que explota a los crédulos en beneficio propio; ni de famoso especialista que acoge la fila de enfermos dispuesto a hacer caja; ni tampoco de voluntario que ofrece un tiempo de ocio sin que afecte a sus seguridades; ni siquiera de político que diseña estrategias de reforma, salvaguardado él mismo de la experiencia de la pobreza.

El así llamado por los especialistas «secreto mesiánico», manifiesto en los pasajes en los que Jesús ordena silencio sobre su identidad a los beneficiarios de su acción sanadora, muestra la voluntad del Señor de centrar su atención sobre el bien del otro. En primer lugar no busca fama sino el bien de la persona en dificultades. Más aún, evita ser malinterpretado como una especie de solucionador inmediato de toda suerte de problemas. Lo cual no quita que el fruto final de la transformación operada consista precisamente en la aceptación de su Señorío, el único que garantiza el bien definitivo de todo hombre.

La compasión de Jesús, como todo su modo de proceder en su vida terrena, está atravesada por el misterio de la Encarnación, tal como lo presenta Pablo en su himno de Flp 2, 4-10. Está sellada por un movimiento combinado de éxodo y de descenso hacia el hombre necesitado de su amor reconstructor. De tal suerte que éste alcanza así por pura gracia a ser constituido en la humanidad del Verbo el «prójimo» de la divina misericordia.

El lenguaje de la compasión en los Sinópticos y en Pablo se relaciona también con el de la piedad, expresado generalmente por el verbo eleeo ${ }^{3}$. Desde este reclamo de afecto seguro al Corazón de Jesús la Iglesia comienza la celebración eucarística, culmen del amor escatológico de la caridad divina actualizada en la historia presente. Con esta humilde invocación (¡Señor, ten piedad!),

2. Mt 9, 2; Mc 2, 5; Lc 5, 20; 7, 48.

3. Mt 5,$7 ; 9,27 ; 15,22 ; 17,15 ; 18,33 ; 20,30 ;$ Mc 5,$19 ; 10,47 ;$ Lc 16,$24 ; 17,13 ; 18,38$; Rom 9, 15; 11, 30; 12, 8; 1 Cor 7, 25; 2 Cor 4, 1; Flp 2, 27; Tim 1, 13.16; 1 Pe 2, 10. 
el creyente y la comunidad se disponen, ostentando humildemente el título de su pobreza reconocida, con la única condición posible, para trasponer el umbral que conduce al abismo sin confines de la divina misericordia.

Resumamos las características del ejercicio de la compasión de Nuestro Señor: 1) Es gratuito. El único título que hace al hombre acreedor de la caridad divina es su pobreza reconocida. 2) Por lo mismo, es un amor misericordioso, desbordante, siempre allende de lo humanamente concebible. 3) Es autoimplicativo. En esto consiste el sello encarnatorio de la acción de Jesús. En su actuación misericordiosa Jesús compromete todas sus posibles ventajas y todos los recursos de su humanidad. De modo que antepone la urgencia de la ayuda, arriesgando su comodidad, interrumpiendo los planes del grupo apostólico, e incluso exponiéndose a una marea tal de incomprensiones que finalmente le acarreará una muerte ignominiosa. 4) Conlleva una correspondencia inmediata a la necesidad del individuo en su concreción histórica. Jesús entra en relación con una humanidad doliente, no a través de informes o de declaraciones, sino en comunión experimentada en su propia carne, expuesta al cansancio, al hambre, al frío y al calor, al desamor, a la soledad, a los oprobios, y finalmente a la muerte física. En esa coparticipación en su propia humanidad de la fatiga existencial del hombre, alivia todo tipo de necesidad impelente, y rescata al hombre de su ominosa caducidad. 5) La acción salvífica de nuestro Señor está siempre orientada a la vocación escatológica de cada hombre. No se detiene en la fruición de las apetencias contingentes histórico-concretas, consideradas como un fin en sí mismas. Abre la percepción creyente del hombre hacia la pregnancia salvífica del sufrimiento, y ofrece el manantial del amor, de la vida y de la libertad, que es Él mismo. Con ello reconduce la andadura del deseo hacia la voluntad de servir, sostiene la fe tambaleante en el infortunio, y permite atisbar el claror de la vida beata en la pena de la hora peregrina.

¿No es precisamente el don de la Eucaristía el resumen de todo ello? En ella el pan cotidiano que quita el hambre aquí y ahora es al mismo tiempo la comunión con el amor definitivo con Aquel que ha vencido el pecado y la muerte.

En el Corazón de Jesús hemos encontrado la alianza y la respectiva conformación de los dos vectores del discernimiento. Aquel que resuena y mana de las entrañas, y aquel que subyuga desde fuera. La firme voluntad del Señor de servir al hombre sin orientación y sin vituallas halla el momento y el modo de volcarse a partir de los reclamos de aquellos a los que va encontrando en el camino.

La actualidad de la caridad divina escanciada en el corazón humano de Jesús constituye para nosotros el arquetipo para entender el modo de inserción y misión del religioso en el mundo. Frente al espejo de este corazón humano de Jesús humilde, apacible, comprometido, misericordioso, operativo, gratuito y acogedor, podemos discernir si nuestro modo de vivir insertados en el mundo refleja al menos un ascua de la ardiente voluntad del Señor de restaurar, curar, enseñar, guiar, consolar y retirar con el perdón el pesado lastre de nuestros pecados. 
Anteriormente nos hemos preguntado desde la fe si el tiempo presente es para nosotros más lugar de promesa que fuente de desgaste. Ahora desde la caridad nos preguntamos si el escenario en el cual se desenvuelve nuestra vida personal y comunitaria es más vaso de misericordia que agarradero de supervivencia. Finalmente desde la esperanza podemos notar si nuestro corazón convertido capta los signos de la emergencia de la novedad del Reino, o es un claustro en el que resuenan solamente lamentaciones.

La vida consagrada en la Iglesia supone la vocación de ser sacramento existencial y comunitario de la caridad de Cristo en su plena dedicación al servicio de los hombres, sobre todo los más necesitados. El Concilio Vaticano II favoreció el título de la caridad perfecta para designar la característica esencial de su identidad. La dimensión de la plena y permanente dedicación a la evangelización, a socorrer las formas más lacerantes de la dignidad humana, vinculada a su expresión comunitaria, constituye parte esencial del significado cristiforme y eclesial de nuestra vocación.

Lo que los fundadores han experimentado al alborear el carisma recibido por la Iglesia a través de ellos es la fascinación del amor de Jesucristo, por encima de cualquier otro vínculo, proyecto o deseo, conjugado con un compromiso hacia los «prójimos» tan apremiante que reclama la plena dedicación en su favor con el concurso de los recursos personales y comunitarios del cuerpo apostólico arracimado en torno a su inspiración.

Ellos vivieron en su momento histórico al modo de Jesús: Afectados por el reclamo de las necesidades de los próximos hasta el punto de poner en pie una comunidad entregada a su ayuda.

No se trata de una característica exclusiva de los llamados carismas apostólicos, sino que forma parte también de aquellos considerados como genuinamente contemplativos. En efecto, así como otros han percibido el reclamo para socorrer a los pobres, a los enfermos, a los marginados de todas las categorías, a los ignorantes o a los increyentes, éstos han sentido con tal fuerza la penuria de una experiencia auténticamente vivida de Dios que han reunido una comunidad que es trasunto de su presencia en medio del afán secular y expresa con su dulce acogida y con su gozosa armonía su invitación para todos a buscar y hallar el Único Necesario.

Por su parte, los llamados por el Espíritu a la cabecera del enfermo, cerca del niño desbandado o del anciano abandonado, arrancados a la comodidad de su ambiente para hacer de los lejanos próximos de su corazón misionero, no son meros asistentes sociales. Éstos están llamados con la calidad de su entrega a testimoniar la fuente superior del amor que les empuja a hacer saborear a otros por si mismos lo que a ellos les ha subyugado, más allá de la resolución más inmediata de sus necesidades.

Algunos se despertaron al servicio a partir de la curación de sus propias heridas, como la esclava Bakkita, convertida después en una emprendedora mujer 
liberadora; otros con las manos en la masa de su compromiso en favor de los demás, como Juan Bosco entre los jóvenes o Juan de Dios entre los enfermos, otros llevados por fuertes intuiciones caritativas más allá del entorno inmediato, como el canónigo Allamano, que sin alejarse del Piamonte formaba sus misioneras en Kenya. Mas todos descubrieron las brechas del dolor y corrieron a restañarlas.

La conformación de la forma específica del reclamo caritativo surgido en el corazón apostólico de los fundadores pertenece a la identidad carismática del Instituto. Ser fieles a esta llamada forma parte de la identidad de nuestros institutos religiosos. Cada Instituto es apostólicamente fértil en la Iglesia en la medida que no pierde de vista la forma específica de caridad contenida en el carisma.

Algunos institutos tienen una finalidad apostólica más abierta y multiforme, otros más orientada hacia una tipología más concreta de sujetos o necesidades. Sin embargo, cada instituto tiene un perfil apostólico determinado desde el carisma fundacional. Esto no significa que a lo largo del tiempo el servicio apostólico deba corresponder a las mismas concreciones socioculturales a las cuales el grupo fundacional dio respuesta. En otras cosas porque en puridad eso es imposible. Vivimos en un mundo sometido a rápidos y fuertes cambios en cada ambiente social. Además, un propósito de mantenimiento literal o fixista del servicio apostólico primero agostaría la creatividad carismática dotada por el Espíritu desde el comienzo y sostenida por Él a lo largo de la historia del instituto. A todos corresponde velar por esa fidelidad creativa distante tanto del inmovilismo cuando de proyectos incoherentes con el propio patrimonio espiritual y apostólico.

Resulta siempre orientativo de nuestra acción preguntarnos qué hubiera hecho el Señor en nuestro puesto y en nuestras circunstancias. Más allá de este analogatum princeps, para preservar el fundamento carismático de nuestra acción apostólica, también puede ayudarnos tratar de intuir qué camino hubieran emprendido nuestros mayores en la presente encrucijada de la historia del Instituto y de la vida de nuestra comunidad. Esa hipótesis sólo produce resultados verosímiles cuando el religioso está imbuido de lo que podríamos llamar un verdadero sensus instituti.

Me parece importante recuperar el modo de vivir y comprender el mundo vivido por los fundadores. Ellos se dejaron concernir por el grito de los pobres, el sufrimiento de los enfermos, el abandono de los ancianos, opacidad ominosa de los descreídos, del abuso de los migrantes, la desorientación de los jóvenes, la insignificancia social de los marginados. Ellos sintieron aquel imperativo amoroso con el que Jesús no permitió a los apóstoles desentenderse de las necesidades perentorias de los que tenían delante.

A mi juicio, una de las tareas principales del gobierno de nuestras instituciones es la de ayudar a recuperar en la conciencia comunitaria del cuerpo apostólico la percepción del «mundo» como escenario de nuestra misión, impulsados por el amor compasivo de Jesucristo según el perfil carismático de cada una. 
Esta conciencia implica una inserción en el mundo apostólicamente inquieta, tendida hacia los reclamos de una humanidad necesitada de cura. Por vocación propia la vida consagrada debería ser la punta de lanza de aquello que el Papa Francisco llama la «Iglesia en salida». Como el mascarón de proa de una Iglesia a la búsqueda de náufragos en un mar proceloso, lejano del puerto de los autosatisfechos.

El despertar de esta sentida y comprometida conciencia apostólica no es fácil. Está amenazada desde diversos frentes. En primer lugar por aquella percepción endogámica que mira lo externo como fuente de seguridades o recurso de supervivencia. Esta tendencia constituye un mecanismo de defensa frente a las dificultades inherentes a la misión. El envejecimiento progresivo de nuestras instituciones, sobre todo en Europa, el agostamiento de nuestros recursos humanos, la dificultad de llevar adelante nuestros proyectos y de hacer culturalmente inteligible nuestro propósito último, pueden reforzar el reflujo de la desilusión, o el desapego afectivo de nuestra misión. Puede hacerse más tentador el repliegue sobre las propias necesidades. Para otros la endogamia puede tener una apariencia más brillante como la búsqueda del prestigio del propio instituto que se quiere socialmente exitoso. Es necesario conjurar esta atmósfera pegajosa endogámica que a veces nos envuelve. Termina por hacernos infecundos.

Sin embargo, la dificultad para encaminar por la senda justa nuestra conciencia apostólica puede venir de un frente más subrepticio. Lo podríamos llamar como una «fuga hacia adelante». Es decir, de un propósito poco maduro de emprender misiones con un cierto glamour social, más impulsado por un voluntarismo ideológico que por un verdadero discernimiento apostólico. Sería como huir a cualquier precio del propio estancamiento, más para otorgarnos una cierta justificación en la medida de lo políticamente correcto, que por acudir a las necesidades que nos reclaman según nuestras reales posibilidades. A veces agitamos al viento slogans que no siempre se corresponden con nuestros reales compromisos personales y comunitarios.

¿Cómo ayudar a recuperar la conciencia apostólica empañada o falta de discernimiento? Entiendo que lo más conducente es beber del mismo manantial de dónde ésta mana. Ir a la misma fuente de donde los fundadores espoleados por la caridad apostólica se han expandido por los caminos del mundo como portadores de dignidad humana y testigos del evangelio. Esta fuente se alimenta de dos canales. El primero y principal es el amor a Jesucristo nuestro Salvador. El segundo está esencialmente vinculado al primero: La disponibilidad compasiva hacia el prójimo.

Uno se pregunta si nuestra conciencia apostólica a veces no se ve reducida por una vida espiritual empobrecida, descuidada por falsas razones, o incluso manipulada desde la búsqueda de seguridades. Una vida en el Espíritu de Jesucristo no puede dejar de ser estimulante e impelente al requerimiento del Señor actuado en el prójimo. Nos conviene recuperar una oración más auténtica, más 
vinculada al verdadero Jesús del Evangelio, que no tiene donde reclinar la cabeza, cuya misión es servir y no ser servido. Por otra parte tenemos que ayudarnos a vivir una vida comunitaria menos blindada, menos atrincherada en rutinas consuetudinarias. Una comunidad más centrada cordialmente en la alteridad de la misión tal cómo ésta se configura en el entorno inmediato. De tal suerte que esta pasión por el bien hacer para los demás sea la que inflame el corazón de sus miembros y la conciencia coparticipada de la comunidad. Bebiendo de esta doble fuente surgirá un discernimiento apostólico más coherente con la voluntad del Señor intuida en los signos del tiempo presente.

\section{ALGUNOS CRITERIOS PARA LAS CONCRECIONES}

En la perspectiva de las concreciones a las cuales nos lleva el discernimiento apostólico, puede ayudar tener en cuenta algunos criterios. El primero sería atender al perfil carismático del compromiso a asumir. Ya lo hemos subrayado. No somos los administradores generales del universo, ni contamos con recursos inexhaustos frente a cualquier necesidad. Tampoco somos una versión reducida de toda la comunidad salvífica que es la Iglesia, sino sólo un humilde miembro de la misma con su misión específica, realizada en el concurso de la gran misión de toda la Iglesia. Nuestra eficacia en el contexto de esa armonía eclesial está en entregarnos con denuedo hacer sonar nuestro instrumento en el concierto de toda la plantilla. El fruto de nuestra inserción eclesial está ligado a la fidelidad de nuestro carisma reactualizado por el Espíritu.

Un criterio que estuvo presente en el despegue apostólico de nuestros Institutos es el de la necesidad. Se trata de la percepción de la urgencia de una ayuda frente a una necesidad, ya sea ésta por su carácter perentorio o difuso, ya sea por la dificultad de otros recursos humanos para afrontarlo. Nuestros Instituto han sido muy solícitos en identificar las bolsas sociales de todo tipo de pobrezas. Y han sido igualmente generosos para emplearse allí donde otros no podían. Estas necesidades cambian según los cambios histórico-sociales. Por lo que el compromiso que surgió en la articulación del primer servicio apostólico está sujeto a un continuo discernimiento para dar una respuesta fiel y creativa en cada lugar de nuestra inserción y en cada etapa de su desarrollo.

El criterio de la necesidad va del brazo no pocas veces de aquél del riesgo $o$ de la dificultad. A veces la necesidad es tanto más impelente cuando se presenta con mayores obstáculos para aliviarla. Pertenece a la naturaleza de la Vida Consagrada, y más concretamente aún de la Vida Religiosa, hacerse presente operativamente en las trincheras más difíciles en las que la Iglesia es más aborrecida o frente a un tipo de acción que requiere presencias más articuladas y sostenidas en el tiempo. La dedicación completa de los religiosos vivida en el espacio y en el tiempo y en la solidez de su tejido comunitario en el que todo se comparte, hace posible estos milagros. En la historia de la Iglesia, en los con- 
textos culturales más arriesgado o reacios, precisamente allí, los religiosos han sido la punta de lanza que poco a poco han abierto camino a la penetración de la Iglesia. Me atrevería a decir que no sólo ha sido, sino que siempre será así. Porque este es el modo en el que se manifiesta la radical vocación pascual de los religiosos, que lleva hasta sus últimas consecuencias la vocación bautismal de todo cristiano.

Pensemos por ejemplo en la evangelización del siglo XX, el siglo de los mártires. En el invierno prolongado de la Rusia colectivista, o de sociedades como la China, sometidas al poder omnímodo del estado, o en los países más afectados por los grupos musulmanes más fanáticos la única forma articulada de la presencia de la Iglesia ha sido la Vida Religiosa. Así como en las sociedades occidentales del bienestar, cada vez más alejadas de los valores trascendentes, la misión de la Vida religiosa ha sido determinante para restañar la brecha entre la fe y la cultura, para despertar a los jóvenes de la manipulación social, y estar cerca de los nuevos pobres completamente fuera del circuito de la asistencia social de los estados.

El carácter eclesial de nuestra misión tiene otra nota de gran alcance. La de su difusión en un perspectiva universal. Sobre todo los Institutos de Derecho Pontificio comparten de modo significativo esta solicitud por las necesidades de la Iglesia universal. Está más vocacionalmente disponibles ya sea a aquella peregrinación apostólica misionera que le arranca continuamente de las seguridades alcanzadas, ya sea porque su tipo de inserción en las Iglesias particulares, sostenida por esta sensibilidad más amplia, evita el enclaustramiento en los interese particulares.

El discernimiento necesario para conjugar los intereses particulares con aquellos más propios de nuestro servicio a la Iglesia universal corresponde sobre todo al gobierno general. Precisamente por la visión supra partes propia del servicio del gobierno general atenta al bien más universal. No es infrecuente que una comunidad religiosa, incluso una provincia, se atrinchere en sus opciones particulares, mohínas frente a una disponibilidad mayor a la perspectiva más amplia del gobierno general. Esto también puede ser fruto de aquel empobrecimiento espiritual al que ya me he referido. Cuando se oscurece nuestro vínculo enamorado con el Señor, nos convertimos en personas más inseguras, y por tanto más aferradas a sus seguridades inmediatas. Eso hace que algunos religiosos no estén dispuestos al cambio frente a las destrezas que han llegado a hacer con el aplauso de la platea, precisamente porque se definen más por lo que hacen que por lo que son.

Quisiera finalmente aludir a un punto que toca el núcleo de nuestra misión en la Iglesia. El de ser maestros y constructores de comunión. Evangelización y comunión son dos aspectos del único misterio de Cristo presente en el misterio de la Iglesia, en tanto que sacramento universal de salvación realizada por el Único Mediador. En este sentido, por una parte la Iglesia universal del envío apostólico. 
Es decir, signo visible e instrumento de la convocatoria universal de los hombres en Cristo, Camino, Verdad y Vida, por medio del envío de las comunidades y de los individuos. No somos, por tanto, una especia de francotiradores que hacen la guerra por su cuenta.

Por otra parte la comunión eclesial es signo visible que anticipa la realidad escatológica de nuestra plenitud de vida en Cristo. La salvación acontece y se instaura como una realidad comunional. El cielo no será nunca un conglomerado de hombres aisladamente satisfechos. Es una comunidad de amor respectivo sin doblez que nos unirá entre nosotros en la fuente misma del amor que es el mismo Señor.

La Vida religiosa no ha nacido como una tropa de individuos dispuestos a actuar en solitario, ni como una masa de gentes teledirigidas. Surgió mirándose en el espejo de la descripción de los sumarios de los Hechos $(2,44 ; 4,32)$, con la férvida voluntad de vivir una unión tal de corazones, que hace ineludible compartirlo todo, biografía, empeño, vida cotidiana, bienes espirituales y materiales.

El individualismo es una de las celadas más peligrosas a las que estamos expuestos. Precisamente porque pone en peligro la identidad crística y eclesial de nuestra vocación. Toca a todos impulsar la dimensión comunional de nuestra vocación. Particularmente en tres dimensiones: En primer lugar garantizando el vínculo eclesial de cada una de nuestras misiones y comunidades por medio del envío en obediencia. Las iniciativas demasiado individualistas, aun cuando fueran brillantes a primera vista, acaban por desmoronarse. Justamente porque se mantienen por la testaruda voluntad de los sujetos, pero no tienen el marchamo de la voluntad del Señor autentificado por la obediencia religiosa.

El segundo criterio de discernimiento apostólico desde la perspectiva de la comunión está ligado a la constitución y mantenimiento de una verdadera comunidad religiosa, incluso en medio de las misiones más comprometidas. La calidad de su vida compartida es signo de la fuerza del evangelio que pretende anunciar, y a la vez es meta comunitaria de la conversión del corazón a la que aboca tal anuncio. En el tiempo de las experimentaciones apostólicas y comunitarias, a veces por la premura de un determinado servicio eclesial se ha podido descuidar un tanto aquellas condiciones necesarias, indispensables en toda comunidad. Condiciones que contemplan un número mínimo de miembros, con una mínima capacidad de entendimiento respectivo, poder contar con un verdadero superior o superiora que garantice la responsabilidad de su marcha, y un ritmo cotidiano que asegure espacios de oración personales y comunitarios, de trabajo y de serena convivencia. La prisa por atender una necesidad urgente descuidando estos mínimos, normalmente no acaba bien, y los frutos que se recogen son exiguos.

El tercer criterio apostólico que afecta a nuestra vocación comunional en la Iglesia se refiere a nuestra capacidad de articular nuestra misión en la comunión con la Iglesia particular y en la Iglesia universal. Esta exigencia hoy es particular- 
mente necesaria. Las dificultades que presenta la nueva evangelización, exige una firme voluntad de todos los miembros de la Iglesia y de todas las comunidades de sumar al esfuerzo común y no de restar. La misión de nuestros Institutos no puede centrarse en la búsqueda de nuestro prestigio, ni siquiera de recoger éxitos inmediatos, sino más bien pretende insertarse en la misión evangelizadora de toda la Iglesia con una grande flexibilidad para cuidar sus flancos más desprotegidos, de cambiar de servicios según las necesidades más apremiantes, e incluso de dejar generosamente a otros lo que con fatiga hemos construido si fuera más conveniente para el conjunto de la misión eclesial. Esta generosa disponibilidad eclesial, sedienta del bien común más que de nuestras propias conveniencias, es bendecida por el Señor con mayores frutos en la perspectiva de la extensión del Reino.

Concluyo con un último reclamo. Entre las necesidades de toda índole que podemos registrar en nuestros prójimos tiene un alcance singular la sed de Dios, gustada, experimentada y plenamente actualizada en el don de nuestra comunión con Jesucristo. La misión de la Iglesia encamina a esta experiencia a la vez fontal y última no por un mensaje recibido de oídas, sino por la comunicación de un testimonio inmediato. Nuestra misión eclesial se funda en el testimonio de un amor absolutamente preferente por Jesucristo. Un amor que empalidece el resto de los vínculos y proyectos que anidan en el corazón humano hasta el punto de estar dispuestos a entregar la vida entera. Un amor servido en bandeja de humilde y alegre caridad a cuantos son llamados por el Señor a experimentarlo por sí mismos en comunión con El. Es decir a todo hombre que viene a este mundo, vocacionado a vivir alojado para siempre en el corazón del Padre, en unión con el Hijo por el don del Espíritu. 